\title{
A Component Architecture for an Extensible, Highly Integrated Context-Aware Computing Infrastructure*
}

\author{
William G. Griswold, Robert Boyer, Steven W. Brown, and Tan Minh Truong \\ Department of Computer Science and Engineering \\ University of California, San Diego \\ La Jolla, CA 92093-0114 \\ E-mail: $\{$ wgg, rboyer, sbrown, mtruong $\}$ @cs.ucsd.edu
}

\begin{abstract}
Ubiquitous context-aware computing systems present several challenges in their construction. Principal among them is the tradeoff between easily providing new contextaware services to users and the tight integration of those services, as demanded by the small form factor of the devices typically found in ubiquitous computing environments. Performance issues further complicate the management of this tradeoff.

Mechanisms have been proposed and toolkits developed for aiding the construction of context-aware systems, but there has been little consideration of how to specialize, organize, and compose these mechanisms to meet the above requirements. We motivate and describe a software architecture that provides the desired integration and extensibility of services in a context-aware application infrastructure. A key result is the fissioning of intuitive class organizations, both across layers and within layers, to achieve the required integration of services and separation of concerns.
\end{abstract}

\section{Introduction}

The small end-user devices found in ubiquitous computing environments place tough requirements on applications. Users want to grasp information quickly, navigate representations fluidly, and respond easily. These requirements drive systems toward tight integration-where every component knows about all other components-in order to simultaneously meet performance expectations and create a composite view that succinctly expresses vital information.

These challenges are critical in a young fast-moving field like context-aware computing. A typical system might regularly undergo the addition of new kinds of context sensors, modeled entity types, services, or end-user devices. To reduce fragile coupling among components, software engineering principles argue for anticipating future changes and introducing separation of the identified concerns. The question, then, is how to achieve separation (e.g., through modularization) without sacrificing too much integration or

* This work is supported in part by an HP Mobility Grant gift, support from the California Institute for Telecommunications and Information Technology (Cal-(IT) ${ }^{2}$ ), and the ActiveWeb project, funded by NSF Research Infrastructure Grant 9802219. To Appear in ICSE 2003. performance. Mechanisms have been proposed and toolkits developed for easing the construction of context-aware systems $[4,10,16]$, but there has been little consideration of how to specialize, organize, and compose these mechanisms to meet the above requirements.

Over the past year and a half we have been building the UCSD ActiveCampus context-aware infrastructure for ubiquitous computing, exploring the challenges of simultaneously supporting extensibility and tight integration. ActiveCampus has been online for a year and now comprises eight integrated end-user services [8]. It is available to everyone at UC San Diego, and is in active use by our extended group. We are now in the process of deploying ActiveCampus to our base of 700 HP Jornada users.

Our experiments with a traditional layered approach [5] combined with a hybrid of the mediator and observer design patterns [15] yielded significant leverage. However, maintaining a high level of integration and performance while maintaining a decoupling of services from modeled entities and from each other required additional architectural constraints and mechanisms. These include stripping the entity object down to a collection of compact context identifiers, employing a service introspection mechanism with an extended service interface, and a scoped, context-indexed data cache. These result in a significant change to the roles of the principal layers in the architecture.

In the following, we first identify the key dimensions of extensibility in a context-aware ubiquitous computing environment, based on our experience with ActiveCampus. These provide a groundwork for the key dimensions of componentization required for an extensible, integrated context-aware computing application infrastructure. Second, we consider current approaches, including the Context Toolkit [4], discussing the extent to which it meets these needs, and what challenges remain. Third, we present our architecture and its evolution to better meet the requirements. Finally, we discuss the implications of this architecture and lay out some open problems.

\section{Background and Motivation}

Building on previous definitions, Dey et al. define context as "any information that can be used to characterize the sit- 
uation of entities (i.e. whether a person, place or object) that are considered relevant to the interaction between a user and an application, including the user and application themselves," where relevance is defined with respect to the user's current task [4]. Context might include, for example, the proximity and availability of colleagues.

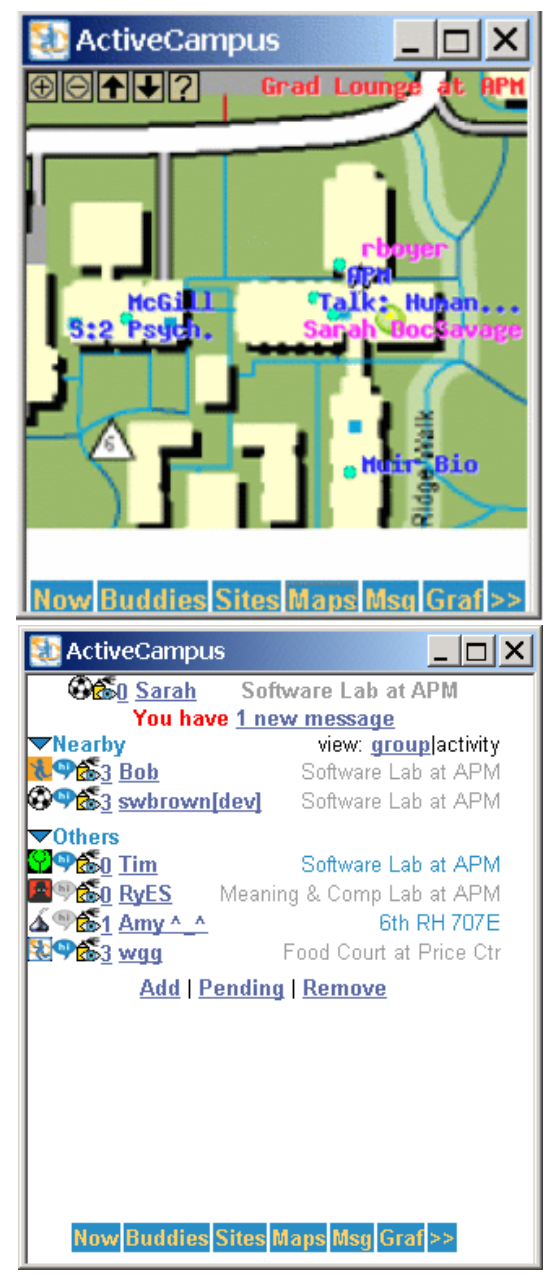

Figure 1. The Map and Buddies services of ActiveCampus. The Map service shows an outdoor or indoor map of the user's vicinity, with buddies, sites, and activities overlaid as links at their location. The Buddies service shows colleagues and their locations, organized by their proximity. Icons to the left of a buddy's name are buttons that show the buddy on the map, send a message to the buddy, and look at graffiti tagged on the buddy. Other services are reached by the button bar or clicking items embedded in the views.

Following Winograd [16], we expand on this definition slightly. Providing a context-aware application through computing devices in a ubiquitous computing setting supposes that the application cum device, as an artifact in the environment, is useful for completing some human-directed task. Hutchins characterizes such artifacts by their ability to efficaciously store, superimpose, and relate multiple representations of information about a task [11]. The artifact then can reduce a person's cognitive effort to recognize important relationships and recall key information, thus permitting the person to allocate unused cognitive resources to other aspects of the task. A classic example artifact is the alidade, which combines a compass, a prism, and a magnifier to adjoin a magnified scene on top of a compass reading, with a single vertical line passing through the center of each. The part of the scene that is bisected by the line is at the heading bisected by the same line. Thus, as compared to using a compass alone, it is possible to take an accurate magnetic heading in the context of visible landmarks with little cognitive effort.

Thus, for the purposes of this paper, we define an enduser context-aware application to constitute the selection, superimposition, and rendering of representations of taskrelevant context, including the superimposition of application operations available for the next step of the task. Because many tasks are emergent and human behavior is often opportunistic, the available actions may include "switches" in the task.

To motivate the dimensions of extensibility and integration required for such an artifact, consider two screenshots of ActiveCampus services (application views) for a user named Sarah, shown in Figure 1. The services show mostly the same information-Sarah's physical proximity to other entities that are present at the time-but in rather different representations. The "task" is to help Sarah, a new student, engage the complex milieu of campus life in the face of obstructions and distractions.

The Map service represents Sarah centered geospatially on a map of her surroundings, with nearby buddies and static entities (e.g., departments) superimposed as well. Once Sarah orients the map to her physical space, she can use this representation to construct a plan for how to reach any of the entities on the map. Clicking on any of her buddies will list a set of services that are available on that buddy (e.g., show map centered on them, send them a message, show digital graffiti on them, show their profile).

The Buddies service also represents Sarah situated with respect to her buddies, distinguishing buddies that are near, as well as their activity level. By showing only buddies and by giving their locations by name, Sarah can conceptualize her active buddies at a relatively high level of detail. If Sarah knows the places by name, she can quickly find her way to a nearby buddy. If not, she can click on her buddy's personal icon to see her buddy centered on the map. The same services available via a click-through on the map are available directly in this service's display, to the left of each buddy name. Also, the area below the title bar is reserved for other services to notify the user of events, like the arrival 
of new messages.

Finally, below both services is a "button bar" of services for one's self, such as editing of privacy settings; these are a superset of the services available on a buddy.

Dimensions of Extensibility. From these two services, we can observe many possible dimensions of extensibility, largely driven by innovation. In particular, someone might want to improve the system by adding one of the following, with the resultant consequences on preexisting components:

- A new service, such as a contextual alarm service [3]. This would require adding the new service to the button bar and giving the alarm service the ability to notify users of triggered alarms through other services. If a user were allowed to add alarms for their buddies ("Hey Brad, check out the 'new books' table at the bookstore!"), then an alarm icon should appear next to each buddy on the Buddies service as well.

- A new kind of context or sensor input format, such as a latitude/longitude representation of location generated by a GPS device. Consumers of location, like the Map service, must be able to cope with locations generated via this device.

- A new kind of modeled physical entity, such as a web cam, which may also come with new kinds of context. Such entities would need to show up in the maps and entity lists of services to show where they are positioned and give access to their functionality.

- A new representation, such as 3-D image of a location. Such representations would have to be correlated to locations reported from sensor devices.

- A new end-user device, such as a pen PDA (personal digital assistant), which uses sound and LED's for output and sound and button clicks for input. Services would need to produce representations that are suitable for 'display' on such a device.

One issue we do not consider is the mobility of a user's work products as the user moves through a heterogeneous computing environment. Aura addresses this issue [13].

Tight Integration. Given the above considerations, it is natural to consider a component architecture that defines a component category for each dimension of extensibility, with standardized interface rules for each category. The interface rules would be defined so that the typical changes under each category could be made behind the component interface, thereby hiding the impacts of those changes from other components in the system.

One challenge in determining such rules is to balance the separation of the component changes against the integration of component functionality. A narrow interface might be easy for a component implementor to satisfy, but might be hard for component integrators to use as wished. Using the Map and Buddies services as examples, we see two immediate needs for tight integration: dense information display and contextual access to one service from another.

Because service representations and actions must often be rendered on devices with compact displays, the information displayed about each entity and service must be customized to fit the exact needs of the current service and limitations of the device. The Buddies service, for example, selectively uses small icons, shortened names, and their careful juxtaposition to maximize the display's utilization. This means that the service must require each of the displayed entities and services to satisfy queries for small icons or short names of themselves and entity locations. Yet, other services benefit from larger icons or full names of entities and locations.

On the Map service, a click on the map shows a set of location-based services available at that location, such as the tagging of digital graffiti. This ability to contextually access one service from another is the very definition of tight integration. Yet, for that integration to be fully realized, a newly introduced service (such as digital graffiti) must be able to insinuate itself into the list of possible actions at that location. If that insinuation is to be modular, then it must be realizable through the Map service's interface, rather than by editing the service's code.

The upshot, is that we need to design an architecture with component contracts that are easy for implementors to satisfy (i.e., supporting innovation), yet whose behaviors are rather open (i.e., enabling integration).

\section{Current Approaches}

The most developed prior work in this area is Dey's Context Toolkit $[1,4]$. To ease the prototyping of a context aware application, it offers a small set of generic 'base' classes from which an application developer can derive applicationspecific classes. These classes are organized around highlevel context-aware application functions: collecting sensor data, combining data from multiple sensors, and translating sensor data into alternate formats. Each of these classes contains all the infrastructure required for distributed peerto-peer storage, communication via XML over HTTP, and software event monitoring:

- Context Widget, accepts and stores context coming from a sensor, abstracting away the details of the sensor. For example, a location widget might gather timestamped locations coming from a GPS device. Context is stored in dynamically typed attribute-value pairs. It provides event notification functionality to signal the arrival of new data. Context widgets also abstract away actions on actuators (e.g., a light switch), and in this case they are called Services. 


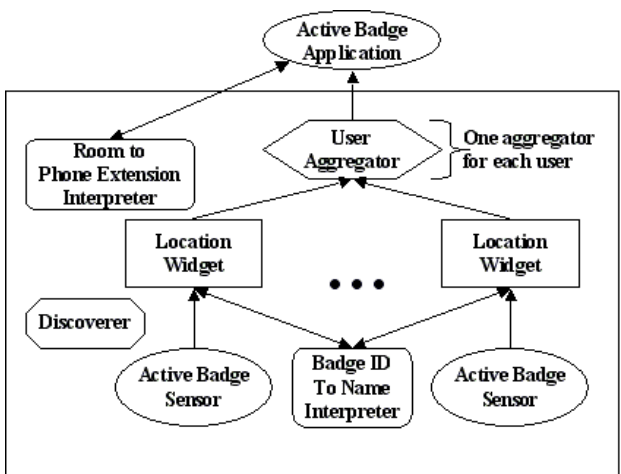

Figure 2. Architecture diagram for the ActiveBadge application using the Context Toolkit [4].

- Context Aggregator, subclassed from Context Widget, aggregates the context of multiple Widgets, and inherits their attribute-value pairs. By listening to events from the Widgets it aggregates, an aggregator can stay aware of changes in the context it monitors.

- Context Interpreter, takes one representation of context and generates an alternate representation, such as taking a GPS reading and producing a map.

- Discoverer, helps applications find one of the above resources by name or capability in the network.

Abstracting away each functional requirement as a class is useful in accommodating key dimensions of change. Yet, as is typical of a toolkit, the Context Toolkit stops short of dictating architectural standards that would enable an application based on the Toolkit to interoperate or integrate with applications of complementary functionality [10]. Indeed, such standards could hinder rapid development. Another concern is that the Toolkit's fine-grained distributed object model entails significant inter-object communication costs and precludes some efficient data organizations, among other issues $[10,16]$.

A key question is how a resource like the Context Toolkit could best be used to define an extensible suite of contextaware applications (what we call services) that share their context-aware underpinnings, their functionality, and even their displays to users. For example, the typical Context Toolkit application contains a number of context widgets, a couple of context aggregators that join those widgets into observable entities (e.g., user), a few context interpreters for the translation and interpretation of context, and an application object that hooks all these up (Figure 2). The application object then interacts with the user objects and context interpreters to produce interactive displays for end users. How then should two applications be fused together? As one possibility, should the user entities in each be merged into a large user object to eliminate redundancy, distributed processes, and event propagation from context widgets? Or should they be left separate, each with their redundant data at risk of becoming inconsistent or causing performance problems? Suppose just one of these applications is used at a time, in sequence, defined by the end user's emergent needs? Is there a way that unnecessary computation from the separately running applications, or the fused application, could be avoided?

In response to questions like these, Hong and Landay advocate a centralized service infrastructure approach [10]. The infrastructure would dictate architectural rules that would ensure that an innovator's component would interoperate with others that hew to the same rules. These rules would include how data and functional responsibilities are allocated to components, how those components relate to each other, and how their interfaces are defined [7, 12].

Along these lines, Hong is developing a Context Specification Language and infrastructure [9]. Winograd proposes a data-centric, two-level blackboard that decouples data from computations [16]. The lower Event Heap tuple-space abstracts away the complexities of coping with ephemeral sensor data. The upper Context Memory level tuple-space supports a persistent object view of the world. In separating computations from data, this proposal shares some of the same motivations and architectural ideas as those presented here, but does not directly address the issue of service integration.

\section{The ActiveCampus Architecture}

As advocated by Hong and Landay, we've taken a centralized client-server approach to providing a context-aware application infrastructure. The ActiveCampus server infrastructure supports all functions that it can provide internally, with only the sensing of context and its rendering to end user devices operating outside the server. This both eases administration of the system and minimizes the requirements placed on sensors and display devices, whose resources for computation and program development tend to be quite limited. Most importantly, centralization provides greater freedom to allocate behavior to components and organize those components to meet the needs of extensibility, integration, and performance. Also, newly deployed services can integrate with and leverage the existing running services.

At the highest-level there are two aspects of the system's component architecture: the interfaces to external components, and the internal system architecture. We briefly discuss the interfaces to external components before discussing the internal architecture.

Sensor-reporting is supported by providing a pair of components connected via XML/SOAP RPC (top two levels of Figure 3, on the left). One component runs on the device to capture and send data, and the other runs on the server to receive the data and marshal the raw data into the 
system database. Both components are typically written in $\mathrm{C}++$. Display to devices (and interaction with them) is componentized similarly, with additional support for HTML transport.

Most of the responsibility for the operation of the context-aware system is managed within the centralized infrastructure, implemented as a web server running PHP and backed by an SQL database. We present our architecture of the centralized infrastructure by first discussing our initial design failures, and then follow with a complete architectural description, as diagrammed in Figure 3.

\subsection{Early Design Experience}

Context-aware computing, especially in a multi-application setting, is both representationally and behaviorally complex. To address these challenges, we settled on a layered design to incrementally provide behavior and assert systemwide properties [5]. The principal layers were, bottom-up: Data Storage (i.e., the database), Data Abstraction (e.g., sensor data and entity objects), Object Correlation (e.g., mapping raw sensor data to internal forms, as well as services themselves), and Environment Proxy (i.e., transport to external devices). To aid the incorporation of new services into ActiveCampus, our initial idea hinged on centralizing the data of entities - the people, departments, devices, and places modeled by the system-in typed entity objects so as to ease their handling by the many services we anticipated incorporating. In particular, sensor data would be accepted and processed into entity objects, and aggregate entity objects would then be the base of data for providing services.

Three problems emerged with this design as services were added to ActiveCampus:

Entity definitions experienced churn and bloat. The evolution of user identity in ActiveCampus is instructive. The identity of a person entity began as a unique internal ID and a login name. Soon a screen name and icon were added. Then the icon was split into a large icon (for clarity) and a small icon (for compactness). Each of these extensions, motivated by a service enhancement, is in fact just adding an alternate representation of the user's identity. Moreover, there is no apparent end in sight; we anticipate adding "earcons" [2] to enable hands-free and eye-free perception of context. From a software design perspective, it is troubling that every service enhancement has entailed enhancements to other objects in the system; the development of services was not being simplified.

Services were not decoupled from each other. Because of the small end-user displays, the fusion of service elements, not just entity data, seemed required to provide concise yet meaningful representations of context. For example, the Buddies service indicates what services are available on buddies, and will notify the user when a new message has arrived. In our initial design, these requirements led to services explicitly calling each other.

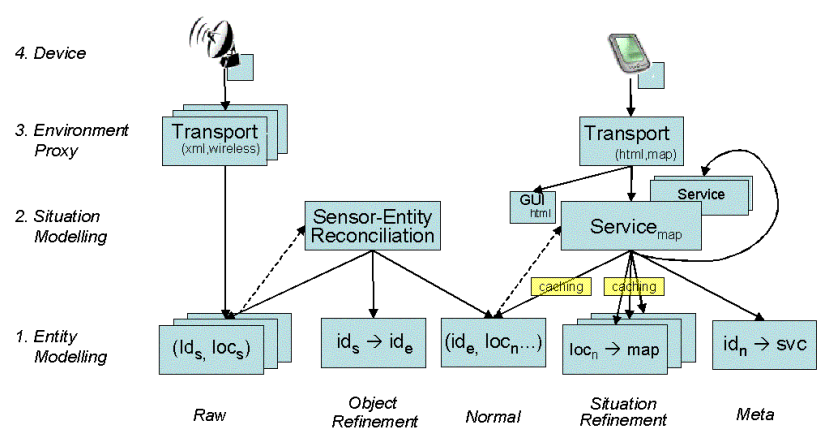

Figure 3. A graphical depiction of the essential elements of the revised ActiveCampus software architecture, focusing on the capture of user location and displaying it on a map.Boxes denote components, solid arrows denote calls, and dashed arrows represent event notifications.

Performance was becoming unacceptable. As more services were added and entity objects grew, database access to entities became a bottleneck. Even if a service was using only a small part of an entity, it had to pay for the bloat introduced by other services. This was exacerbated by services calling each other, which resulted in re-querying entity data. Naive efforts at reducing re-querying by data sharing increased the coupling of services through the sharing.

\subsection{Internal Component Architecture}

Our analysis of these problems resulted in the architecture shown in Figure 3. There are five layers, top to bottom, the last not shown. Boxes denote components, solid arrows denote calls, and dashed arrows represent event notifications. We briefly outline the architecture here, and then further motivate and elaborate the architecture with respect to the three observed problems:

4. Device: In the top Device layer, components run on devices and connect to ActiveCampus through RPC.

3. Environment Proxy: The Proxy layer marshals data between Devices and ActiveCampus's internals.

2. Situation Modeling: The Situation Modeling layer synthesizes the situation of an individual entity from multiple available data sources. For example, it translates quirky sensor representations to internal forms and associates them with entities. It also assembles and superimposes contextual representations of entities for end-user display (i.e., service execution). From the Context Toolkit's point of view, these are Context Interpreters.

1. Entity Modeling: The Entity Modeling layer represents entities (e.g., users and sensors) in several forms: raw external representations (e.g., a dynamically assigned network address), fast-indexing normal forms (e.g., a 
permanently assigned unique integer), and forms for presentation (e.g., a screen name). It also represents the static relationships among them. From the Context Toolkit's point of view, the components are (left to right) Context Widgets, Context Interpreters, Context Aggregators, and Context Interpreters.

0. Data (not shown): The data layer provides for efficient storage and retrieval of data, in our case through an SQL database. The data layer may store data differently than apparently organized in the Entity Modeling layer for reasons of performance.

We point out three salient features of this architecture before going into the deeper discussion. One, a key feature of this architecture is how the representations of the Entity Modeling layer are integrated by the Situation Modeling layer, using a hybrid mediator-observer design pattern [6, 15]. Two, introspective mechanisms are used by services to refer to each other generically, thereby avoiding explicit service coupling. Three, caching mechanisms interposed between the Entity Modeling and Situation Modeling layers cope with performance problems.

\subsubsection{Entity Bloat: The Entity and Situation Layers}

Our first mistake was to confuse an entity's intrinsic data with the presentation of that data. Consider again entity identity. In database design or object-oriented design, an entity can be uniquely identified with an integer or object reference. The other forms of 'identity' discussed above, like screen name, are merely alternate representations (i.e., presentations) of that identity. Being alternate rather than intrinsic representations (and since their introduction is driven by the enhancement of services rather than entities), they can be associated to the entity through separate relations that are referenced through the unique identifier. These alternate representations are not necessarily unique (e.g., multiple persons may have the same screen name). The same observation applies for all intrinsic entity data, especially data that can be represented in multiple ways, such as locations.

Entity Normalization. Following through with this insight entails the following architectural rules for representing entities relating entities to other data:

- Only data that is intrinsic to an entity is part of its object. Alternate representations of intrinsic data should be stored separately.

- Intrinsic data should be represented in a compact normal form that is suitable for use as an index for fast retrieval of alternate representations.

- Any kind of context (e.g., location) should be standardized into an indexable "normal form" representation as well. For example, all location indices are required to be $\{x, y, z\}$ tuples with a fixed origin, orientation, and distance scale. Any representation to be retrieved using that kind of context should be indexed by its standardized index representation. For example, maps should be directly retrievable with normal-form location tuples. Thus, any location, regardless of its source, can be used to retrieve data from any locationindexed representation.

- All associations (mappings) of context (e.g., buddy membership, $\left.\left(i d_{1}, i d_{2}\right)\right)$ should be represented using their indexed forms. The association of alternate representations (e.g., (Sarah, Brad)) should be reserved only for final display. ${ }^{1}$

With these rules, adding a new service has no impact on an entity. For example, a service that requires the addition of earcons results in adding a new component that maps between entities and earcons. The independence of both the representation and its mapping from the service makes it reusable by other services without further coupling services. Moreover, it is now trivial to translate from a user to a location to a map, as each is guaranteed to be indexed against standardized indexed representations.

Entity and Situation Modeling. Raw sensor data, by its nature, is not in entity-normal form. It is a "pre-entity" abstraction of entities until translated into a normal form and re-stored as entities. For example, the network MAC address of a wireless device may be used to identify it in the system until it can be converted into an internal system ID.

The pre-normal status of sensor data is true in another way as well: although a device itself is a modeled system entity, it is also used for computing the context information for another entity, like a person, who is only loosely associated with the device. For example, a person may carry a wireless PDA, whose wireless signals can be used for geolocating the person. However, it is all too easy for the person to leave the PDA in the office when going to lunch. Yet, the cell station providing connectivity for the cell phone in the person's pocket may continue to generate legitimate data about the their location.

Such sensor data must be processed in light of other context data to derive a probably-correct entity-centric interpretation. This indeed is what our Object Correlation layer had done, although with a simple one-to-one mapping that did not allow for many-to-many mapping of context to entities.

Now that we have distinguished both the presentation forms and the pre-entity sensor forms of an entity from its normal form, it becomes apparent that the Data Abstraction layer is really an Entity Modeling layer, providing these

\footnotetext{
${ }^{1}$ Although an object's identity is classically represented by the object reference itself, we use a unique integer ID suitable for database queries. This could be hidden from applications through abstraction, but we have chosen not to do so.
} 
three dimensions of entity modeling. Also, because of the complexity of mapping first from raw sensor data to normalform entities, and then from entities to their rich presentation by services, it is appropriate to reconceptualize our Object Correlation layer. We rename it the Situation Modeling layer to signify its role of processing data from multiple entities in a context to discern or present the situation of an entity.

The complex relationship between these three forms of entity data deserve special architectural attention. Even though the states of entities are derived from sensor data, entities have no direct dependence on them or the processing of their data. For example, new forms of sensing or sensor processing should have no direct impact on entities. Applying design patterns to this problem suggests a hybrid mediator-observer design that manages the sensorentity relationship through an independently operating component $[6,15]$. In our case, the Sensor-Entity Reconciliation component, operating as a third party, mediates the complex sensor-entity relationship by receiving change notifications from the sensor components and propagating updates to the appropriate entities. This separation protects entities from implementation changes in the sensor domain. This pattern is also applied in relating entities to the views generated by services; here, services themselves are the mediators between entities and views.

In contrast to this separation, the Context Toolkit's structure encourages Context Aggregators (e.g., entities) to directly connect to Context Widgets (to aggregate them). The translation of Widget data for use by an Aggregator is performed by a Context Interpreter that is called by either the Widget or the Aggregator. Context Interpreters are simple passive input-output translators without the native capability to function as a mediator between Widgets and Aggregators.

\subsubsection{Service Coupling: Introspective Interaction}

The decoupling of entities and services does not do away with the service coupling observed earlier. Using the Buddies service as an example, the question is how the service could come to know, with low inter-service coupling, (a) what services are available on a user's buddy, (b) what services want to notify the user of something, and (c) how to render service activators and notifications on its view?

To help decouple services, we employ a registration mechanism, along with interface rules for services to follow so the mechanism can work:

Service Structure. All services are designated to run on behalf of an entity, usually a user, called the subject. Most services also can be run on the accessible context of an entity different than the subject, known as the object. For example, if the Maps service is run by Sarah on Brad, she will see the map view centered on Brad, rather than herself. ${ }^{2}$

To support inter-service integration, all services objects are required to support the following methods:

boolean compatible(id subj, \&optional id obj)

id renderID(id subj, \&optional id obj)

The compatibility method is a type check to determine whether the types of the subject and object can support the successful execution of the service (e.g., they are of subtype Locatable and hence provide a location).

The renderID method indicates how to render the target service's activation button, without specifying the representation of that button (i.e., keeping entity references in normal form). For example, the renderID call on the Map service returns the object's (buddy's) ID if object is bound. In the Buddies service, the buddy's map service is indicated by the buddy's small icon (but other services might wish to render the Map service as text). Thus, the Buddies service passes the renderID result to the smallicon mapping component to retrieve the icon. As a way of providing state information about the service, a call on render ID might even return an ID for a state (e.g., "Available") rather than the ID for the service, subject, or object.

Active services must also provide an implementation of this method:

id msgWaiting(id subject, \&optional id object)

which lets a rendering service test whether the active service has a notification for the subject. If so, the method returns a non-null entity ID for the notification that can be rendered a number of ways, like the ID returned from renderID. This test permits general notifications when object is omitted, and object-specific notifications when object is provided (e.g., new messages from object).

Registration. When a top-level service comes online, it is registered as available and is assigned a unique entity identifier by the system. If the service needs to be run regularly to notify users of important information, it also registers itself as an active service. Additionally, the developer of the service will provide values for the representations of a service's activation button: short name, icon, and tooltip.

Introspection. To render the services for an entity, a rendering service $R$ iterates over the registered services $S$. For each service $S_{i}, R$ calls $S_{i}$ 's compatible method with the invoking user as the subject and the entity (e.g., buddy) as the object. For those calls returning true, $R$ calls $S_{i}$ 's renderID method with the same arguments. $R$ then uses the returned ID to obtain a representation of $S_{i}$ 's activation button. ${ }^{3}$

\footnotetext{
${ }^{2}$ The object could be a set of entities, but the need for this has not yet emerged, in part because the subject's own context includes associated entities like buddies that serve as the implied objects of the service.

${ }^{3}$ For developers who want absolute control over the rendering of their service's display, they might introspect on the available services merely to detect that a new one has been added and notify the developers.
} 
Similarly, $R$ can iterate over all active services with waiting messages, $A_{i}$, using $A_{i}$ 's msgWaiting method to get an ID for use in rendering $A_{i}$ 's notification.

\subsubsection{Speed: Scoped, Context-Indexed Caching}

Decoupling services and unbundling intrinsic entity data from renderable representations of entities potentially compromises performance. A service like Buddies queries large amounts of entity data itself, then delegates to passive services for rendering of the buddies, and finally checks with active services for notifications. Many of these checks on other services use data already retrieved by the top-level service. However, to minimize service coupling, this data is not part of the inter-service interfaces. To address this problem, the ActiveCampus infrastructure provides a simple two-level caching mechanism.

To implement this mechanism, we take advantage of the fact that ActiveCampus applications can tolerate inconsistent and stale data. As an example of tolerable inconsistency, it is not critical that a person's location and their buddy's location be reported for the exact same point in time. As an example of tolerable staleness, a person's location from 10 seconds ago is a reasonable approximation of their location now. Data that has minimal tolerance for inconsistency and staleness (e.g., passwords) is not cached.

Caching is achieved through the memoization of method calls into the Entity Modeling layer. To bound the staleness of Normal Entity data, we scope their memoization to the lifetime of a top-level call to a service. If the service itself causes changes to data (e.g., the user is manually asserting a change in their location), the service is responsible for updating the cache.

Because the renderable representations of entities are relatively stable and have weak consistency requirements (e.g., the change of a buddy's icon is not critical), they may be cached across an entire user session (e.g., login). Because this data is indexed by its normal form, efficient lookup is possible.

\section{Discussion}

We have designed a context-aware software architecture around the criteria of extensibility, integration, and performance. Surprisingly, moving away from the Context Toolkit's focus on rapid prototyping causes several aspects that may appear in a single Toolkit object to be dispersed across several components in ActiveCampus. The dispersion both spans layers and occurs within a layer. In the following, we discuss this outcome and its consequences.

Fissioning Objects across Layers. Employing the traditional architectural themes of layering of abstractions and mediation of representations implies a rather faceted design in contrast to the Context Toolkit. In particular, we spread its "built-in" functions of abstraction, distribution, and storage across three different layers. This allows exclusion of unneeded elements, independent control of the implementation of each aspect, and assigning the development of each to an expert (e.g., a database specialist) who can integrate their functions over many objects if needed (e.g., database table organization).

This separation also permits the placement of other components in between. Most notably, we found it beneficial to place context interpreters in layer 2 of the system, above data abstraction but below distribution. Moving this functionality above distribution would have hurt performance, and of course moving below abstraction would have exposed too many representation details. Theoretically, this decoupling should also permit us to "move the line" that separates the internal and external components of ActiveCampus, giving external agents greater control, computational responsibility, and the ability to provide services. For example, a service component could be pushed above the proxy line by providing a pair of proxy components that handles the service's calls to the internal architecture. To be successful, however, there would be performance and consistency issues to be resolved.

Intralayer Fissioning of Modeled Entities. Other than the fusion of abstraction, storage, and distribution, the Context Toolkit's class design is relatively neutral about how classes should be combined into an application. One architectural rule of the Context Toolkit is that Context Aggregators are a subclass of Context Widgets. This particular insight is represented in our architecture by placing our sensor representations and other entities in the same layer of the architecture. However, we have added several additional architectural rules to meet our goals of extensibility, integration, and performance. First, we stripped our entity objects of any presentation data, instead using normal-form indices to represent entity context that can be used to index into presentation representations. This leaves raw sensor data in a pre-entity form and presentation data in a entityindependent form.

Intralayer Fissioning of Situation Modeling. The consistency of the various forms of entity representation is maintained by the Situation Modeling layer, and driven by events outside the system. Interestingly, Dey et al. have explored the addition of a situation abstraction to the Context Toolkit [4]. What we observe here is that achieving consistency across views of an entity requires analysis of an entity's situation. Thus, sensor-entity reconciliation is both a context interpreter and a situation abstraction. This fusion of functions and roles in a single object suggests that packaging an abstraction like "situation" in an object could be problematic. "Context interpretation" is a basic system function that can be reused in a number of ways. However, this particular context interpreter fulfills the role of pre-entity situation analysis. Likewise, an ActiveCampus service also performs situation modeling for rendering ef- 
ficacious views to end-users for performing their own situation analysis. In some sense our services are "aggregated interpreters", fulfilling the Context Interpreter function in the same way that Context Aggregators fulfill the Context Widget function.

What we see then is that the Situation Abstraction that Dey and Abowd write about is more of a system-wide role fulfilled by a layer, rather than a reusable abstraction (although we note that the facade design pattern is a layerlike object [6, p. 185]). In particular, the situation of an entity is two stages of interpretation by the Situation Modeling layer. The U.S. Department of Defense's data fusion paradigm suggests that are at least five levels (really phases) of situation modeling [14]:

0. Sub-Object Data Association and Estimation: signallevel data association and characterization.

1. Object Refinement: combines data from multiple sensors and other sources to determine position, kinematics, and other attributes.

2. Situation Refinement: develops interpretation of the relationships among the objects and events in the context of the operational environment.

3. Significance Estimation: intent prediction and consequence prediction.

4. Process Refinement: monitors the fusion process to refine the process itself and guide acquisition of additional data.

To a first approximation, our two situation modeling components in Figure 3 perform fusion levels $(0,1)$ and 2 , with an end-user performing the last two levels with the assistance of the displays produced by level 2 .

Given ActiveCampus's Entity and Situation Modeling layers, with refinement moving to the right, enhancing ActiveCampus with significance estimation modeling would involve adding two new columns on the right side of the architecture. In particular, services would store analyzable versions of their superimposed representations to a new Entity Situation component in the Entity Modeling layer, which would be consumed by new Significance Estimation components in the Situation Modeling layer.

Situation Elaboration via Inter-service Introspection. Rich situation modeling requires services to communicate with each other. Given a mechanism that can generically enumerate the available services, one way to avoid a complete fusion of such services is a generic inter-service interface that allows one service to render small amounts of information for other services, or perhaps even surrender control. For this purpose we identified the key service metafunctions of: (a) checking compatibility with the entities on which they might be run, (b) providing hints as to how to render their activators, and (c) notifying entities of important context information. Normal-form entity identifiers play a key role in fulfilling the separation, as they enable services to communicate in terms of representation-neutral identifiers rather than a particular presentation that may be inappropriate to the rendering of the top-level service.

Future Work. This architecture has not been fully tested. It has been successful in decoupling existing services, but new ones have yet to be added. The ultimate success of the architecture will hinge on whether new entities and services manifest the characteristics of interaction we've seen in the past, or instead manifest unanticipated ones.

One dimension of change we have not addressed to date is the addition of radically new device types. ActiveCampus, for the most part, has taken a lowest-commondenominator approach to the rendering of services, assuming that all devices can render text and small images straightforwardly via web browsers. Advanced javascripting techniques had to be avoided because the PDA web browsers provided inconsistent levels of support. ActiveCampus responds to the standard device pragmas that browsers provide, but not in a sophisticated way. Someone who wished to use ActiveCampus through a device such as a pen PDA, which used sound and LED's for output and sound and button clicks for input, would be disappointed.

Supporting a radically new device-without creating a duplicate set of services for it-might be handled by designing services around a strategy pattern [6, p. 315] that is parameterized by the renderable representations. A service then could be created by a factory [6, p. 107] that instantiates the service's representation strategy according to the device's output characteristics.

\section{Conclusion}

The construction of a ubiquitous context-aware computing system faces the difficult tradeoff between the tight integration of application services and the ease with which they can be added to the system. Performance considerations further complicate the management of tradeoffs.

We have motivated and described a software architecture that provides the necessary separation of concerns for the above considerations in a context-aware application infrastructure, and reported on our use of this architecture in the construction of the ActiveCampus system. We found that a hybrid mediator-observer design pattern structured by layering provides significant leverage. However, maintaining a high level of integration and performance while maintaining a decoupling of services from modeled entities and other services requires additional architectural constraints and mechanisms. These include stripping the entity object representation down to a collection of context index identifiers, employing a service introspection mechanism with additional rules for service interfaces, and a scoped, context-indexed data cache. 


\section{Acknowledgements}

We thank Jeremy Weir, Jolene Truong, Tim Foley, Neil McCurdy, David Harbottle, R. Benjamin Shapiro, Greg Jay, Rommel Pesigan, David Hutches, Jadine Yee, Justin Lee, Steve Lau, Daniel Wittmer, Antje Petzold, Jean Aw, Linchi Tang, Joseph Chow, Adriene Jenik, Jim Hollan, and Jason Chen for their help. We thank UCSD's Facilities office, especially Roger Andersen, Robert Clossin, and Kirk Belles, for their expertise and resources. We thank Intel's Network Equipment Division for donating network processors and Symbol Technologies for their technical support.

\section{References}

[1] K. D. A. Providing Architectural Support for Building Context-Aware Applications. $\mathrm{PhD}$ thesis, Georgia Institute of Technology, College of Computing, December 2000.

[2] M. Blattner, D. Sumikawa, and R. Greenberg. Earcons and icons: Their structure and common design principles. Human-Computer Interaction, 4(1):11-44, 1989.

[3] A. K. Dey and G. D. Abowd. Cybreminder: A contextaware system for supporting reminders. In Intl. Symposium on Handheld and Ubiquitous Computing (HUC'O0), pages 172-186, 2000.

[4] A. K. Dey, D. Salber, and G. D. Abowd. A conceptual framework and a toolkit for supporting the rapid prototyping of context-aware applications. Human-Computer Interaction, 16(2-4):97-166, 2001.

[5] E. W. Dijkstra. The structure of the "THE"multiprogramming system. Communications of the ACM, 11(5):341-346, May 1968.

[6] E. Gamma, R. Helm, J. Vlissides, and R. E. Johnson. Design Patterns: Elements of Reusable Object-Oriented Software. Addison-Wesley, Reading, MA, 1995.

[7] D. Garlan and M. Shaw. An introduction to software architecture. In V. Ambriola and G. Tortora, editors, Advances in Software Engineering and Knowledge Engineering, volume 1, pages 1-39. World Scientific Publishing Company, Singapore, 1993.

[8] W. G. Griswold, R. Boyer, S. W. Brown, T. M. Truong, E. Bhasker, G. R. Jay, and R. B. Shapiro. Activecampus sustaining educational communities through mobile technology. Technical Report CS2002-0714, UC San Diego, Department of CSE, July 2002.

[9] J. I. Hong. Context fabric: Infrastructure support for contextaware systems. Qualifying Exam Proposal, 2001.

[10] J. I. Hong and J. A. Landay. An infrastructure approach to context-aware computing. Human-Computer Interaction, 16(2-4):287-303, 2001.

[11] E. Hutchins. Cognition in the Wild. MIT Press, Cambridge, 1995.

[12] D. E. Perry and A. L. Wolf. Foundations for the study of software architecture. SIGSOFT Software Engineering Notes, 17(4):40-52, October 1992.

[13] J. P. Sousa and D. Garlan. Aura: an architectural framework for user mobility in ubiquitous computing environments. In 3rd Working IEEE/IFIP Conference on Software Architecture, 2002.
[14] A. N. Steinberg, C. L. Bowman, and F. E. White. Revisions to the JDL data fusion model. In Proceedings of SPIE AeroSense (Sensor Fusion: Architectures, Algorithms and Applications III), pages 430-441, Orlando, Florida, 1999.

[15] K. J. Sullivan and D. Notkin. Reconciling environment integration and component independence. In Proceedings of the SIGSOFT '90 Fourth Symposium on Software Development Environments, pages 22-33, December 1990.

[16] T. Winograd. Architectures for context. Human-Computer Interaction, 16(2-4):401-419, 2001. 